\section{Delayed neuropsychiatric syndrome after carbon monoxide poisoning: inclusion of hyperbaric oxygen therapy in the recovery protocol}

\author{
Dante Lo Pardo, Davide Amedola, \\ Giuliana Senatore, Alberto Damiano, \\ Gabriela Pezzuti, Nicola Pugliese, \\ Gianpiero Locatelli, Alfredo Siani, \\ Nicola Maria Vitola \\ San Giovanni di Dio e Ruggi d'Aragona \\ University Hospital, Salerno, Italy
}

\begin{abstract}
The delayed neuropsychiatric syndrome can arise in the period from 4 days to 5 weeks following carbon monoxide poisoning, and is characterized by neuropsychological deficits, which in some cases become chronic. This case report describes an adult female who apparently suffered self-inflicted carbon monoxide poisoning. She was not treated with hyperbaric oxygen and developed delayed sequelae on day 20 . The treatment started with 40 sessions of hyperbaric oxygen therapy and subsequently with neuropsychological rehabilitation and physiotherapy. The treatment resulted in a progressive and almost complete physical and psychological recovery as evidenced by neuropsychometric tests and diagnostic imaging performed in the follow-up. Recovery was attributed to hyperbaric oxygen therapy. Although further research is required, we propose hyperbaric oxygen therapy also in the recovery protocol in the treatment of delayed neuropsychiatric syndrome.
\end{abstract}

\section{Introduction}

Carbon monoxide (CO) poisoning has been a preferred method of suicide ${ }^{1}$ due to its high success rate of approximately $30 \%$. C0 toxicity is the result of a combination of tissue hypoxia-ischemia through the formation of carboxyhemoglobin ( $\mathrm{COHb})$ and direct $\mathrm{CO}$ mediated damage at a cellular level. ${ }^{2-4}$

The spectrum of symptoms depends on the duration of the exposure and the levels of CO.,3 The brain and the heart are most susceptible to $\mathrm{CO}$ toxicity. In the central nervous system $\mathrm{CO}$ causes platelet to neutrophil aggregation, the release of myeloperoxidase and proteases leading and oxidative damage that may explain the clinical syndrome of delayed neurological damage..$^{2-4}$
Timely diagnosis of $\mathrm{CO}$ poisoning is critical because the clinical presentation is non-specific. Treatment consists of the patient's removal from the source oxygen exposure and the immediate administration of $100 \%$ highflow supplemental oxygen and supportive measures if indicated. ${ }^{1,2}$ After an episode of CO poisoning lasting from 4 days to $4-5$ weeks, ${ }^{4}$ delayed neuropsychiatric sequelae (DNS) can appear. The predictors for the development of DNS following acute CO poisoning include: serious consciousness disturbance at emergency admission, head findings indicating hypoxic encephalopathy at computed tomography (CT), hematology findings including high creatine kinase, creatine kinase-MB (CK-MB) and lactate dehydrogenase levels. ${ }^{5,6}$

When DNS appears, the most frequent symptoms are sudden headache attacks, impaired concentration, short-term memory loss, comprehension difficulties, personality change, dementia, limb rigidity with cogwheel sign, bowel incontinence. ${ }^{7,8}$ It is still unclear why such devastating cerebral damage can occur some weeks after the actual episode and what role can be precisely played by the hyperbaric oxygen therapy (HBOT) in preventing DNS. ${ }^{9}$

HBOT aims both to control the clinical manifestations during the acute phase and to prevent delayed neurological damage. ${ }^{10,11}$ A number of studies assessed the incidence of DNS, which is reported to be between 23 to $76 \%$. However, intoxicated subjects treated with appropriate HBOT were not included. ${ }^{9-11}$ The pathogenesis may be ascribable to some autoimmune processes, which can result in severe neurological dysfunction. ${ }^{12}$

CO poisoning requires a specific follow-up program. Neuropsychometric tests and diagnostic imaging are the examinations of choice in the assessment of delayed neuropsychiatric sequelae. ${ }^{13}$

\section{Case Report}

This case report describes an adult female who apparently suffered self-inflicted carbon monoxide poisoning. She had a serious consciousness disturbance at emergency admission. Cerebral CT showed no areas of altered density in the brain and other predictors for the development of DNS following acute CO poisoning were negative. The arterial blood gas test reported a low value of $\mathrm{COHb}$, yet the staff did not consider it significant, since the patient was reported to be a heavy smoker. Electrocardiography was within the limit, with the absence of QT abnormality. Toxicological urine tests were negative for opiates, cannabinoids, amphetamine, cocaine, benzodiazepines and the blood test reported no pres-
Correspondence: Nicola M. Vitola, San Giovanni di Dio e Ruggi d'Aragona University Hospital, Largo Città di Ippocrate, 84131 Salerno, Italy. Tel: +39.089 .672142 .

E-mail: nicola.vitola@gmail.com

Key words: Carbon monoxide poisoning; Hyperbaric oxygen therapy; Delayed neuropsychiatric sequelae; Suicide attempt.

Received for publication: 9 December 2015.

Revision received: 22 March 2016.

Accepted for publication: 24 March 2016

This work is licensed under a Creative Commons Attribution 4.0 License (by-nc 4.0).

CCopyright D. Lo Pardo et al., 2016

Licensee PAGEPress, Italy

Emergency Care Journal 2016; 12:5675

doi:10.4081/ecj.2016.5675

ence of alcohol. Other laboratory tests such as blood urea nitrogen to creatinine ratio, lactic acid, and arterial blood gas analysis were within the limits. For the cardiac enzymes, CK and CK-MB were normal, while the troponin-I was slightly increased to an initial laboratory test and promptly returned to normal in a subsequent monitoring.

Due to the persistent comatose state, the patient - breathing spontaneously - was admitted to the intensive care unit and treated with oxygen therapy with a Venturi mask at $40 \%$.

After two days, the patient regained consciousness, and was transferred to the Psychiatric Unit in good psychological condition, despite some signs of underlying depression. She appeared calm, cooperative, lucid and oriented, although occasionally a little slow. The patient was not administered any drug during her stay in the Psychiatric Unit.

Around the $20^{\text {th }}$ day of her stay in the Psychiatric Unit, and after a period of relative well-being, the patient began to manifest various clinical symptoms, such as semi-confused state with slowness, impaired short-term memory, agnosia, apraxia, distractibility, tangential, indecision and overt cognitive impairment. Her speech tended to be disorganized. She had bowel incontinence and spastic spasms mainly in the lower limbs with clumsiness, uncertainty in the upright position and uncoordinated movements. This set of symptoms was consistent with the onset of a DNS.

Following the onset of DNS, a treatment was started with multiple sessions of HBOT. ${ }^{14-19}$ In particular, HBOT treatment consisted of 40 sessions in a hyperbaric chamber at 2.5 ATA for 80 minutes with the following sequence: once a day for 18 days, then 10 sessions once every second day and subsequently 8 sessions twice a week and 4 more sessions once a week. 
Since the first sessions, a steady improvement of clinical conditions was registered, from both a physical and psychological perspective. It was accompanied to a gradual recovery of serious cognitive deficits, which permitted the following neuropsychological and physiotherapy approach.

By the $15^{\text {th }}$ session, the behavioral and clinical assessments started to show good adaptation to everyday life. All of the previous problems gradually disappeared: from the notable spastic contracture of the lower limbs, the almost total bowel incontinence, the inability to take care of her own daily dietary and physical needs, to the psychological and relational difficulties which, to say the least, had put the patient in conflict with her surrounding environment. Therefore, her personal interactions could then be described as normal.

So, during following interviews conducted in the Psychiatric Unit, we learned about the obsessive-compulsive behavior of the patient, with a clear desire to self-harm, due to incompatibility with her family environment. We were also informed that she attempted suicide by inhaling the exhaust fumes of her car late in the evening, two days before she was taken to the hospital, therefore after about $40 \mathrm{~h}$.

The imaging results confirmed the gradual improvement of the clinical condition. The electroencephalography (EEG) carried out at the onset of DNS reported a slow electrical activity, while it showed a normal activity by the end of hyperbaric therapy.

Brain magnetic resonance imaging (MRI) (Figure 1) showed initially an acute hypoxic ischemic damage characterized by diffuse signal changes in periventricular, sub-cortical and centrum semiovale white matter along with frontal diffusion (diffusion weighted imaging; DWI) restriction changes. There was no accumulation in the basal ganglia with the gradient-echo technique.

The subsequent follow up after 28 HBOT applications showed a significant improvement with reduced hyperintensity in the previously described area of the white matter and the disappearance of the frontal diffusion (DWI) restriction changes. After the end of the HBOT applications, the MRI showed a further improvement in signal changes as well as in diffusion tensor imaging (DTI). This technique shows the tractographic details of the white matter fibers (Figure 2), which appear particularly damaged in the anterior portion of the centrum semiovale, while cortico-spinal tracts, inter-emispheric tracts (corpus callosum) and parietal regions are sufficiently preserved.

The data was confirmed by fractional anisotropy (FA) results, radial diffusion (RD), and mean diffusion (MD). In particular, we observed a decrease in FA and a relative increase in $\mathrm{RD}$ and $\mathrm{MD}$, which gradually stabi- lized along with the clinical improvement of the patient.

Also the psychopathological and neurological profile gradually improved until the time of discharge, when the patient appeared calm, lucid and oriented within normal parameters, and able to relate to others.

Psychometric tests (Table 1), carried out by the psychiatric unit showed a progressive improvement. The mini-mental state examination score based on age and years of education rose by 4.31 points, from 23.08 at the onset of DNS to a final score of 27.31 after 40 sessions of HBOT. The 15-word Rey test reported a progressive improvement of long-term memory, from a raw score of 3 in the initial immediate recall to a score within the normal standard limits for long-term memory, both in immediate and deferred recall.

Finally, from an ineffective performance of executive functions assessed by the frontal assessment battery, the patient attained a score within the normal range by the end of hyperbaric treatment.

\section{Discussion}

Indication of HBOT is controversial. A 2008 clinical policy statement by American College of Emergency Physicians concluded that HBOT

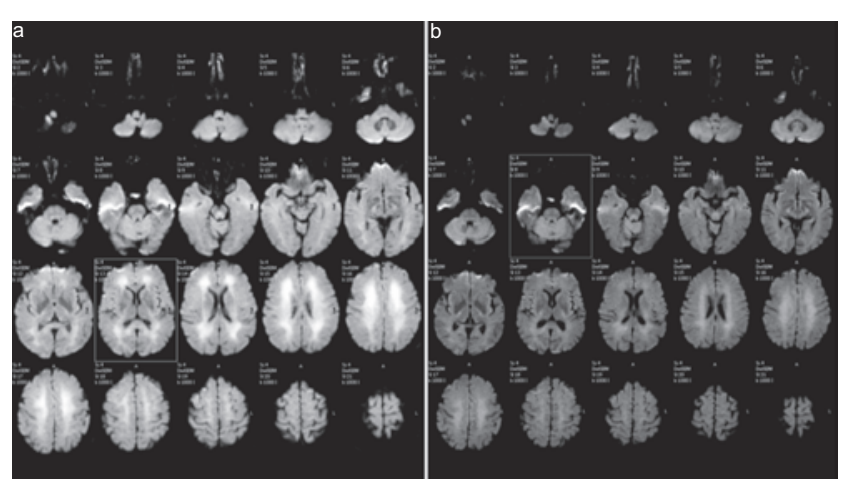

Figure 1. Brain magnetic resonance imaging results at the onset of delayed neuropsychiatric syndrome (a) and after 28 sessions of hyperbaric oxygen therapy (b).

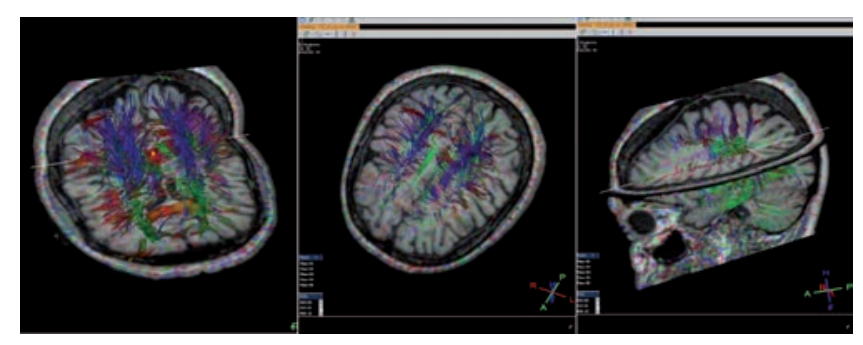

Figure 2. Diffusion tensor imaging taken at the end of the hyperbaric oxygen therapy.

Table 1. Psychometric tests used for follow up of delayed neuropsychiatric sequelae.

\begin{tabular}{|c|c|c|c|c|c|}
\hline & & & e/equival & & \\
\hline & I & II & III & IV & V \\
\hline MMSE & $6 / 4.31$ & $13 / 11.31$ & 21/19.31 & $29 / 27.31$ & 29/27.31 \\
\hline FAB & - & - & - & $10 / 0$ & $15 / 1$ \\
\hline Rey word test immediate recall & - & $3 / 0$ & - & $29 / 0$ & $55 / 4$ \\
\hline Rey word test deferred recall & - & & - & $8 / 2$ & $10 / 3$ \\
\hline
\end{tabular}

I, at onset of DNS; DNS, delayed neuropsychiatric sequelae; II, after 22 sessions of hyperbaric oxygen therapy; III, after 28 sessions of hyperbaric oxygen therapy; IV, after 32 sessions of hyperbaric oxygen therapy; V, after 40 sessions of hyperbaric oxygen therapy; MMSE, mini-mental state examination; FAB, frontal assessment battery. 
is a therapeutic option for CO poisoned patients. Howewer, its use could not be mandated. A Cochrane review after evaluating 6 randomized controlled trials concluded that existing evidence does not establish whether the administration of HBOT for CO poisoning reduces the incidence of adverse neurologic outcomes. Comatose patients with acute nonsuidical $\mathrm{CO}$ poisoning and high levels of $\mathrm{COHb}$ may be the most suitable candidates. The use of HBOT is controversial and if used should be relegated for those patients presenting with severe symptoms, high $\mathrm{COHb}$ levels, or pregnancy. The clinical course of the DNS after CO poisoning is characterized by prolonged hospital stay and a higher number of HBOT sessions. However, there is no specific treatment in patients with DNS, therefore treatment will be challengeable.

The present case report suggests that HBOT decreases the severity of impairment in patients with DNS. Although a large randomized trial is required to address the efficacy of this therapy, therapeutic application of HBOT may be recommended in patients with DNS after $\mathrm{CO}$ poisoning.

HBOT has a positive effect on DNS because induces cerebral vasoconstriction with reduction in cerebral edema and results in more rapid dissociation of $\mathrm{CO}$ from respiratory cytochromes. $^{20}$

\section{Conclusions}

The case report in this work suggests that HBOT decreases the severity of impairment in patients with DNS. To evaluate the validity of the indices of the clinical effect of HBOT, we performed brain MRI, EEG and neurocognitive tests. Our results showed that changes in EEG signals and neurocognitive tests were closely correlated with the patient's clinical course. Although further studies are required to evaluate conclusively the efficacy of HBOT, this therapy may be suggested in DNS after carbon monoxide poisoning.

\section{References}

1. Hitosugi M. Suicide due to carbon monoxide poisoning-trends and preventive measures. Rinsho Byori 2008;141:40-4.

2. Wolf SJ. Clinical policy critical issued in the management of adult patients presenting to the ED with acute carbon monoxide poisoning. Ann Emerg Med 2008;51:13852 .

3. Hampson NB, Piantadosi CA, Thom SR, et al. Practice recommendations in the diagnosis, management, and prevention of carbon monoxide poisoning. Am J Resp Crit Care 2012;186:1095-10.

4. Vezzani G. La sindrome post-intervallare: ipotesi patogenetica. Med Sub Iperb 2007;2:68-70.

5. Kudo K. Predictors for delayed encephalopathy following acute carbon monoxide poisoning. BMC Emerg Med 2014;14:3.

6. Pepe G, Castelli M, Nazerian P, et al. Delayed neuropsychological sequelae after carbon monoxide poisoning: predictive risk factors in the Emergency Department. A retrospective study. Scand J Trauma Resusc Emerg Med 2011;19:16.

7. Katirci Y, Kandis H, Aslan S, et al. Neuropsychiatric disorders and risk factors in carbon monoxide intoxication. Toxicol Ind Health 2011;27:397-406.

8. Borras L, Constant E, De Timary P, et al. Long-term psychiatric consequences of carbon monoxide poisoning: a case report and literature review. Rev Med Intern 2009;30:43-8.

9. Thom SR, Taber RL, Mendiquren II, et al. Delayed neuropsychologic sequelae after carbon monoxide poisoning: prevention by treatment with hyperbaric oxygen. Ann Emerg Med 1995;25:474-80.

10. Buckley NA. Hyperbaric oxygen for carbon monoxide poisoning. Cochrane Database Syst Rev 2011;4:CD002041.

11. Weaver LK, Valentine KJ, Hopkins RO. Carbon monoxide poisoning: risk factors for cognitive sequelae and the role of hyperbaric oxygen. Am J Resp Crit Care 2007;176:491-7.

12. Petrolini V, Bigi S, Vecchio S, et al. Il monossido di carbonio: "killer silenzioso" e "grande imitatore" (II parte). Emerg Care J 2008;2:20-6.

13. Locatelli C. Intossicazione acuta da monossido di carbonio: il punto di vista del tossicologo. Med Sub Iperb 2009;2:19-23.

14. Watanuki T, Matsubara T, Higuchi N, et al. Clinical examination of 3 patients with delayed neuropsychiatric encephalopathy induced by carbon monoxide poisoning, who recovered from severe neurocognitive impairment by repetitive hyperbaric oxygen therapy. Seishin Shinkeigaku Zasshi 2014;116:659-69.

15. Chang DC, Lee JT, Lo CP, et al. Hyperbaric oxygen ameliorates delayed neuropsychiatric syndrome of carbon monoxide poisoning. Undersea Hyperbar M 2010;37:2333.

16. Lee HF, Mak SC, Chi CS, et al. Hyperbaric oxygen for carbon monoxide poisoninginduced delayed neuropsychiatric sequelae. Zhonghua Yi Xue Za Zhi (Taipei) 2001;64:310-4.

17. Mizuno Y, Sakurai Y, Sugimoto I, et al. Delayed leukoencephalopathy after carbon monoxide poisoning presenting as subacute dementia. Internal Med 2014;53:14415.

18. Spagnolo F, Costa M, Impellizzeri M, et al. Delayed hyperbaric oxygen treatment after acute carbon monoxide poisoning. J Neurol 2011;258:1553-4.

19. Oh S, Choi SC. Acute carbon monoxide poisoning and delayed neurological sequelae: a potential neuroprotection bundle therapy. Neural Regen Res 2015;10:36-8.

20. Neubauer RA, Neubauer V, Ko Chi Nu A, et al. Treatment of late neurologic sequelae of carbon monoxide poisoning with hyperbaric oxygenation: a case series. J Am Phys Surg 2006;11:56-9. 